\title{
Inter-organizational Networks as Patterns for Self-organizing Multiagent Systems
}

\author{
Tore Knabe ${ }^{*}$ \\ German Research Center for \\ Artificial Intelligence (DFKI) \\ Stuhlsatzenhausweg 3 \\ 66123 Saarbrücken, Germany \\ tokn@dfki.de
}

\author{
Michael Schillo \\ German Research Center for \\ Artificial Intelligence (DFKI) \\ Stuhlsatzenhausweg 3 \\ 66123 Saarbrücken, Germany \\ schillo@virtosphere.de
}

\author{
Klaus Fischer \\ German Research Center for \\ Artificial Intelligence (DFKI) \\ Stuhlsatzenhausweg 3 \\ 66123 Saarbrücken, Germany \\ kuf@dfki.de
}

\begin{abstract}
Market-based approaches for task-assignment multiagent systems consist of customer agents with jobs to assign, and provider agents that have the resources to perform these jobs. Jobs can be complex in the sense that they require the collaboration of several provider agents. We present a set of sociological forms of inter-organizational networks that have the potential to increase performance through the structure they impose on collaboration. This gain of structure is especially valuable in settings where communication is limited, which is an appropriate assumption in large-scale applications. We empirically evaluate these organizational forms according to the amount of communication required and the rate of failed task-assignments, and compare them to a system without organizational forms. Furthermore, we investigate the effect of letting agents choose at runtime in which kind of organizational form to engage and which other agents to choose for this collaboration. Our evaluation shows that the proposed organizational forms and mechanisms for selforganization have the ability to improve the efficiency of a market-based multiagent system.
\end{abstract}

\section{Categories and Subject Descriptors}

I.2.11 [Computing Methodologies]: Artificial IntelligenceDistributed AI, Multiagent Systems

\section{General Terms}

Algorithms, performance, reliability.

\section{Keywords}

Organizational Modeling; Self-Organization; Task-Assignment

*This research was funded by the Deutsche Forschungsgemeinschaft in the priority program Socionics under contract Si 372/9-2.

Permission to make digital or hard copies of all or part of this work for personal or classroom use is granted without fee provided that copies are not made or distributed for profit or commercial advantage and that copies bear this notice and the full citation on the first page. To copy otherwise, to republish, to post on servers or to redistribute to lists, requires prior specific permission and/or a fee.

Copyright 2002 ACM X-XXXXX-XX-X/XX/XX ...\$5.00.

\section{INTRODUCTION}

The setting for this paper is a market of customer and provider agents. The customer agents have jobs they need to be done by the providers. These jobs may require more types of resources than a single agent can provide, hence providers need to collaborate. If the same type of job needs to be assigned several times or some parts of changing jobs are constant, the system might be more efficient if providers who are successful at completing a task or subtask together form organizations: relationships that facilitate long-term teamwork. To model these joint activities, the concept holon is used [1]. To the outside, each holon is represented by a distinguished head (agent) which moderates the activities of the holon. Apart from the head, each holon consist of a set of body agents.

We propose a number of such holonic forms motivated by inter-organizational networks found in human societies (Section 2) and investigate their properties in the described task-assignment scenario. Pre-designing the organizational structure is often not possible because the designer does not know which demand will arise during run-time. We therefore also investigate how the agents can decide how to organize at run-time. Providers have to decide when to build new organizations, with whom to build them, and how to adapt (Section 3). We tested our model empirically with respect to two different performance measures (Section 4).

\section{ORGANIZATIONAL NETWORKS}

We describe five different types of holonic organization for MAS. In general, we allow agents to be members in several organizations at the same time. In order to tell which of their organizations is responsible for an incoming order, this rule is limited to the case that all organizations were created for producing different types of jobs, hence for any job the apropriate organization can be unambiguously determined.

As the basis for task-assignment we apply the Holonic Contract Net with Confirmation Protocol (HCNCP) [2]. It is used for inter-organizational communication, market interaction, and intra-organizational communication in the organizational form virtual enterprise. For a more detailed description of the organizational forms cf. [3].

In the market-style interaction, agents directly exchange jobs and some kind of utility (in human society: money).

In a virtual enterprise (VE), there is no single head designated in advance. A VE is product-specific. Each member 
may accept jobs, but must start a new internal HCNCP auction for each of its subtypes among its partners.

Strategic networks (SNs) consist of a head and body agents. If an incoming order matches the product of the SN, the rules applying to the receiving agent depend on whether it is the head or a body agent. Body agents may not directly accept an order from outside, but must bounce it, meaning that they refuse it, but send the name of their head inside the refusal message so the sender of the order can resend it to the head. Heads can accept orders from outside. They know about their body agents' schedules and resources, and can instruct them to do a task at a given time. SNs are product-specific, so multiple memberships are allowed.

Members of a group are not allowed to be members of any other organization. Any incoming order has to be processed as group. Body agents have to bounce incoming orders. Head agents may order body agents to do a specific task.

We model corporations by letting the head assimilate the resources of its body agents. After the assimilation, the body agents are removed from the simulation. The head then acts like a single agent, except that it does not form new organizations.

\section{SELF-ORGANIZATION}

Agents decide with whom to build an organization by keeping a record of the trade history with other agents. The decision process rides on the normal auction process: whenever a customer has found a group of agents that together can complete its job, this group checks whether they should form an organization.

We only let new organizations form if the agents participating in the completion of the job are not acting in the agenda of an existing organization. The decision of whether to build a new organization is based on an algorithm working on a graph. The nodes of the graph are the agents that take part in the completion of the customer's order. Each agent in this graph checks its total trade volume with the other agents and selects those whose trade volume exceeds a given threshold. The agents selected this way are connected to the agent with an edge in the graph. If the graph is connected, the agents agree to form a new organization.

If agents decide to build an organization as described above, they form a VE. This organizational form can be upgraded to a form with more commitment if the collaboration in the organization has shown to be profitable for the agents. VEs can be upgraded to SN, SNs to groups, and those to corporations. All organizational forms except the corporation can be resolved. Agents who are part of a group that decides to form a corporation merge into a single agent and remain so for the rest of the simulation.

Deciding whether to keep, upgrade, or resolve an existing organization is made by voting, based on the average volume per round of orders the agents have processed via this organization. If this average volume exceeds an agent-specific threshold, the agent votes for upgrade. If it is below another threshold, the agent votes for resolve. If all agents vote for upgrade, the organization tries to upgrade. If at least one agent votes for resolve, it is resolved.

\section{EXPERIMENTAL RESULTS}

We investigate two kinds of settings: settings with and without self-organization. In the first kind, we investigate organizational forms separately under the same conditions. In the second, we let the agents start out as market agents and compare a setting where they are allowed to self-organize to one where they are not. We evaluate the system by measuring the total number of messages used in the auctions and the rate of failed jobs.

In scenarios without self-organization, where only one organizational form is used, the number of messages for market relationships is significantly higher than for the other organizational forms. It is higher for the VE than for the $\mathrm{SN}$, which in turn is higher than that for the group. Corporation scenarios have the lowest number of messages. The explanation for this is that the VE is based on a full internal HCNCP, whereas the SN protocol leaves out the proposal phase, the group protocol the proposal and the confirmation phase, and the corporation all internal communication.

The rate of failed jobs is significantly higher for market agents than for the other organizational forms. The VE is the least successful of the non-market organizational forms, but still much better than market agents. The efficiency of the SN comes next. This increase of efficiency might be explained by the reduced protocol; unlike the VE, the SN does not need to collect proposals from its body agents.

In scenarios with no organizations formed in advance, the number of messages is significantly lower when selforganization is allowed. The rate of failed jobs in the system is slightly above 50 percent in scenarios with and without self-organization. Self-organization does not seem to provide the expected reduction in the rate of failed jobs.

\section{CONCLUSION}

We improved the performance of task-assignment multiagent systems by organizing agents in different forms of holonic agents and letting the agents create and change their structure of organization depending on the situation. The organizational forms are inspired by a sociological description of inter-organizational networks. Whether to pre-design the organizations or use self-organization depends on the specific conditions of the scenario: if the order situation does not change and it is important to have a low rate of failed task-assignments and few messages used for the auctions, it is advisable to make all organizations either groups or corporations. If the order situation does change, then agents should start in a pure market relationship context and be allowed to self-organize. Our investigation showed that this will reduce the number of messages in the system significantly.

\section{REFERENCES}

[1] K. Fischer, M. Schillo, and J. Siekmann. Holonic multiagent systems: A foundation for the organization of multiagent systems. In Proceedings of the First International Conference on Applications of Holonic and Multiagent Systems (HoloMAS'03), In print.

[2] T. Knabe, M. Schillo, and K. Fischer. Improvements to the FIPA contract net protocol for performance increase and cascading applications. In International Workshop for Multi-Agent Interoperability at the German Conference on AI (KI-2002), 2002.

[3] M. Schillo. Self-organization and adjustable autonomy: Two sides of the same medal? Connection Science, 14(4):345-359, 2003. 